\title{
Exact Solution for a Chainlike Cluster Growth Model
}

\author{
Zsolt Gulácsi \\ Department of Theoretical Physics, Lajos Kossuth University, H-4010 Debrecen, P.O. Box 5, Hungary \\ Miklós Gulácsi \\ Theoretical Division, Los Alamos National Laboratory, Los Alamos, New Mexico 87545
}

(Received 21 December 1993)

\begin{abstract}
We present an exact solution for a cluster growth model, describing chainlike histories with ordered bond measure and external constraint connected to maximum bond length. We analyze in detail the physical applications that are connected to the parameter regions, where the dominant interparticle interactions are of short-range type.

PACS numbers: 61.46.+w , 02.30.Lt, 05.45.+b, 36.40.+d
\end{abstract}

The clusterization process is one of the most complex and fundamental problems of disordered systems, and as such it continuously attracts interest in a large spectrum of fields such as statistical physics, nucleus and atoms [1], molecular physics [2], astrophysics [3], solid state physics [4] including metallic systems [5], insulators [6], and amorphous systems [7]. The thermodynamic behavior of a disordered system is determined by the fact that its constituent elements are isolated from one another or they form groups of neighbors, called clusters. Systems where the clusterization processes are dominant are governed by the size of the clusters. Much attention has been given to cases where the cluster size is comparable with the lattice size, and the formation of an infinite cluster in the system is imminent, leading to percolation. However, less attention has been given to understanding the processes which generate carbon [8], spin [9], or hard dipolar sphere [10] clusters containing only few components and their physical properties. This is the focus of our study.

The intensive study of carbon clusters [8] was motivated by its importance in various fields such as fullerenes aggregation from gas phase [11], astrophysics [3], and combustion processes [8]. The ${ }^{12} \mathrm{C} /{ }^{13} \mathrm{C}$ isotope scrambling measurements [12] show that the carbon cluster grown in gas phase initiates from an atomic carbon level from where the fullerene formation begins $[2,11]$. Under the conditions of temperatures below [13] $4000 \mathrm{~K} \sim$ $0.34 \mathrm{eV}$, carbon gas density under $\sim 10^{-3} \mathrm{~g} / \mathrm{cm}^{3}$ and no carrier or buffer gas present [14], chainlike structures were measured [15-18] up to a number of elements, nine, and an average cluster size [19-22] around three. The chain formations are also confirmed by molecular dynamics [7], where the calculated average coordination number is 2 at low densities. For our purpose, it is important to emphasize that the interparticle potential is of short-range type [23], and the equilibrium bond breaking energy [7,15] is $\sim 3 \mathrm{eV} \gg k_{B} T$, i.e., the clusters are growing in shortrange conditions [24]. They are chemically stable up to $\sim 9$ atoms $[8,13]$ which are mobile within a cluster and the bonds will attain $\sim 1.3 \AA$, the interatomic equilibrium distance. Similar clusters were also detected in the circumstellar absorption spectrum of the ICR +10216 carbon star [3] and shown [7] to exist in liquid carbon at low $\left(1 \mathrm{~g} / \mathrm{cm}^{3}\right)$ densities.

Similar clusters also appear in spin systems, e.g., in insulating $\left(\mathrm{Eu}_{x} \mathrm{Sr}_{1-x}\right) \mathrm{S}$ at [9] $x \ll 0.13$ (the percolation threshold). The spins of the Eu ions will form few component clusters [6]. The spin-spin interaction is of a superexchange type, vanishing after the sixth neighboring distance [25]. Therefore, in this disordered spin system, similar to the previous carbon gas case, the clusterization process is governed firstly by short-range conditions [24] and secondly by the thermal excitation acting as an external bond breaking process. Studies of the liquid-gas transition [26] in systems of hard dipolar spheres [10], with application in ferrofluids [27], phase transitions [26] also show similar clusterization effects at high $T$ and low density, governed by the same short-range conditions and an external bond cutting energy $[10,28]$. It is evident that the short-range conditions are generic to all disordered systems, in which few component chainlike clusters are dominant. That is, the clusterization process must be governed by a unique statistical process, independent of the system in which it occurs.

The existence of a generic cluster law, for given conditions, can also be reflected at a mathematical level. We define the cluster growth problem in a standard manner: A history is a sequence $h=\left\{s_{i}\right\}$ of $M$ space positions of some elements $\mathcal{E}=\left\{E_{i}\right\}, i \leq M-1$, where $h$ is a connected set, called cluster of mass $M$. The set $H\left(C_{M}\right)$ has various possible histories with a property $H$ that leads to the cluster $C_{M}$. If $\eta$ is an external constraint, connected to the clusterization process, our task is to find for the well-defined and fixed $(H, \eta)$ conditions the probability $p\left(C_{M}, \eta\right)$ that satisfies $1=$ $\sum_{M} p\left(C_{M}, \eta\right)=\sum_{M} \sum_{H\left(C_{M}\right)} p(h, \eta)$, thus explaining the presence of the $C_{M}$ clusters within the system. The calculation of $p\left(C_{M}, \eta\right)$, within the frame of $(H, \eta)$, is normally handled by numerical simulations starting from a relatively low [29] $M$, and the existence of clusters 
is a priori assumed without an in situ analysis. We, however, calculate $p\left(C_{M}, \eta\right)$ analytically and present an exact solution.

Based on experimental observations, the disordered system is composed of elements randomly distributed in a three-dimensional space, in a low concentration of $n_{e}$ with an average interelement distance $r_{0}$. The elements interact through short-range type interactions $W[m(i, j)]$, with an effective interaction length $l$, where $m(i, j)$ denotes adjoining bond measures. Fixing the interaction to be of a short range, we have defined the $H$ property which leads to the formation of the clusters. This means that connections between adjoining elements can occur if and only if the condition $m(i-1, i) \geq m(i, i+1)$ is satisfied. Consequently, the condition constrains the coordination number to 2 , and, accordingly, the formed $C_{M}$ clusters are of chain type. In order to obtain a cluster growth model, a constraint governing the bond breaking has to be considered. This is nothing other than the external constrain $\eta$, mentioned earlier. The way to do this is to consider a bond cutting energy $\eta=E_{c}$ with the property to break a bond if $|W[m(i, j)]| \leq E_{c}$. That is, $\eta$ acts to fix the maximum $m(i, j)$ as $m_{\mathcal{M}}=\max [m(i, j)]=f(\eta)$. As the experimental data suggest, often $E_{c}=k_{B} T$, thus a temperature dependent cluster growth process appears. In this case, usually $m_{\mathcal{M}}$ is a decreasing function of $T$, $m_{\mathcal{M}} \rightarrow 0$ corresponding to $T \rightarrow \infty$.

In this Letter, we will analyze the above mentioned clusterization process in situ, based on concrete mathematical principles. Accordingly, the idea of a generic clusterization law connecting together the presented large spectrum of phenomenon from astrophysics to spin systems is indeed possible. Our claim is that by fixing the $(H, \eta)$ key parameters as presented above the cluster growth model becomes exactly soluble, being the first exact solution in this field. This represents a vital source of information due to the lack of similar (exactly soluble) theories for other cluster growth models.

In order to obtain $p(h, \eta)$ with the property $H$, we built up a cluster by adding element after element, deducing $p\left(C_{M}, \eta\right)$ via integration with cutoff in $r$, as follows. The probability that an element has no neighbors within a sphere of radius $r$ is the well known [30] form $P_{0}(r)=\exp (-\alpha)$, where $\alpha=(4 / 3) \pi n_{e} r^{3}$. The probability that an element does exist in a spherical shell [30] between $(r, r+d r)$ is $d P(r)=n_{e} d v=3 \alpha d r / r$. Thus, the probability that a given element has its nearest neighbor between $r$ and $r+d r$ is $P_{1}(r) d r=P_{0}(r) d P(r)=3 \alpha(d r / r) \exp (-\alpha)$. This is the history probability for one element, which integrated (because of $\eta$ ) up to $m_{\mathcal{M}}$ will give $p\left(C_{M=1}, \eta\right)=\exp \left(-\alpha^{\prime}\right), \quad \alpha^{\prime}=\alpha\left(r=m_{\mathcal{M}}\right)$. The probability of having a two component cluster is obtained as follows: The probability that a given element $E_{0}$ has its nearest neighbor $E_{1}$ between $r$ and $r+d r$ is again $P_{0}(r) d P(r)$. Though $E_{1}$ has no neighbors at a distance less then $r$. Accordingly, the history probability becomes $P_{2}(r) d r=P_{0}(r)(d P) P_{0}(r)$, obtaining $P_{2}(r) d r=3 \alpha(d r / r) \exp (-2 \alpha)$. Integrating over $r$ up to $m_{\mathcal{M}}$, we find $p\left(C_{M=2}, \eta\right)=1 / 2-\exp \left(-2 \alpha^{\prime}\right) / 2$. Considering a cluster of three components, the probability that an element $E_{0}$ has its nearest neighbor $E_{1}$ between $r_{1}$ and $r_{1}+d r_{1}, E_{1}$ has its nearest neighbor $E_{2}$ between $r_{2}$ and $r_{2}+d r_{2}, r_{1} \geq r_{2}$, but $E_{2}$ has no other neighbors at a distance less then $r_{2}$, is $\quad P_{3}\left(r_{1}, r_{2}\right) d r_{1} d r_{2}=P_{0}\left(r_{1}\right) d P\left(r_{1}\right) P_{0}\left(r_{2}\right) d P\left(r_{2}\right) P_{0}\left(r_{2}\right)$. Integrated over $\int_{0}^{m_{\mathcal{M}}} d r_{1} \int_{0}^{r_{1}} d r_{2}, \quad$ it is obtained $p\left(C_{M=3}, \eta\right)=1 / 3-\exp \left(-\alpha^{\prime}\right) / 2+\exp \left(-3 \alpha^{\prime}\right) / 6$. The above procedure can be easily generalized for $M>3$. The history probability for fixed $M$ is given as

$$
\begin{aligned}
p(h, \eta)= & \prod_{i=1}^{M-1}\left\{P_{0}[m(i-1, i)] d P[m(i-1, i)]\right\} \\
& \times P_{0}[m(M-2, M-1)] .
\end{aligned}
$$

The $i$ th term in the main product expresses the fact that $E_{i-1}$ has no neighbors in a sphere of radius $m(i-$ $1, i)$ and has a counterpart in a spherical shell $[m(i-$ $1, i), m(i-1, i)+d m(i-1, i)]$. The last $P_{0}$ in Eq. (1) defines and ends the sequence of the cluster, fixing the number of components to $M$. To obtain the final probability, we integrate Eq. (1) over fixed histories as

$$
\int_{H\left(C_{M}\right)}=\int_{0}^{m_{\mathcal{M}}} d m(0,1) \int_{0}^{m(0,1)} d m(1,2) \ldots \int_{0}^{m(i-2, i-1)} d m(i-1, i) \ldots \int_{0}^{m(M-3, M-2)} d m(M-2, M-1),
$$

obtaining

$$
P_{M} \equiv p\left(C_{M}, \eta\right)=\frac{(-1)^{M+1}}{M !} \mathcal{K}^{M}+\left(1-\delta_{M, 1}\right) \sum_{j=0}^{M-2} \frac{(-1)^{j}}{j !} A_{M-2-j} \mathcal{K}^{j}, M \geq 1,
$$

where the equation $A_{i}=\frac{(-1)^{t}}{(i+2) !}+\left(1-\delta_{i, 0}\right) \sum_{j=1}^{i} \frac{(-1)^{j+1}}{j !} \times$ $A_{i-j} \quad i \geq 0, \quad \mathcal{K}=\exp \left(-\alpha^{\prime}\right)$ and $\quad \sum_{i=0}^{\infty} A_{i}=1$. It can be shown that $P_{M} \geq 0$ and $\sum_{M} P_{M}=\sum_{i=0}^{\infty} A_{i}+$ $\left(1-\sum_{i=0}^{\infty} A_{i}\right) \sum_{j=1}^{\infty}\left[(-1)^{j+1} / j !\right] \mathcal{K}^{j} \equiv 1 \quad$ automatically holds. Furthermore, for fixed $m_{\mathcal{M}}$, in $M \rightarrow \infty$ limit, $P_{M}$ decreases to zero.
With the knowledge of $P_{M}$, different configurational averages can be calculated exactly. For example, the average elements in the cluster $\bar{M}=\sum_{M=1}^{\infty} M P_{M}=e \exp (-\mathcal{K})$ equals $e=2.718$ for $m_{\mathcal{M}} \rightarrow \infty$ and 1 for $m_{\mathcal{M}} \rightarrow 0$. In many experiments the $\eta \equiv k_{B} T$ condition holds, in which case at $T \longrightarrow 0, P_{M}=A_{M-2}$, and $P_{M=0}=0$ [31]. The 
susceptibility $\chi$ of a random spin system can also be determined exactly using $\chi=\left(n_{e} / 3 T\right) \sum_{M=1}^{\infty} P_{M} \overline{S_{M}}\left(\overline{S_{M}}+1\right)$, where $\overline{S_{M}}$ is the average number of spins within a cluster. In the case of spin systems [6], $J(r)$ is a strongly decreasing function [25,32]. By direct calculation, considering Ising spins, we obtain $\overline{S_{M}}=S \int_{r_{0}}^{m_{M}} d r_{1} \mid 1+$ $\sum_{n=1}^{m}\left[\prod_{i=1}^{i=n} \operatorname{sgn} J\left(r_{i}\right)\right] \mid \times \prod_{i=2}^{i=m}\left[\int_{r_{0}}^{r_{1}-1} d r_{i} P_{1}\left(r_{i}\right)\right], \quad$ where $r_{0}=a / \sqrt{2}$ is the lowest possible distance between the Eu ions, $\operatorname{sgn} J(r)$ is given in Ref. [25], and $\mathcal{K}$ is deduced using the experimental data [25] $\mathcal{K}=\exp \left[-0.00275 \ln ^{3}(6.93 / T)\right]$ [32]. From the susceptibility we can calculate the Curie constant, $C=3 T \chi / n_{e}$, to be $C / C_{x}=\sum_{M=1}^{\infty} B_{M} P_{M}$. The $T$ dependence of the Curie constant $C$, normed to its high temperature value $\left(C_{\infty}\right)$, is compared to the experimental data [6] of $\left(\mathrm{Eu}_{x} \mathrm{Sr}_{1-\mathrm{x}}\right) \mathrm{S}$ at concentration $x=0.025$ in Fig. 1. For any given concentration, the calculated expression of the Curie constant is exact. In Fig. 1 we have $x=0.025$, this determines the $B_{M}$ coefficients exactly (e.g., $B_{1}=1$, $B_{2}=0.2768$, and $B_{3}=0.1366$ ). Accordingly, the theoretical curve from Fig. 1 contains no fitting parameters. The clear match between the measured $C / C_{\infty}$ and our theory is proof that our model explains accurately the behavior of the $\left(\mathrm{Eu}_{x} \mathrm{Sr}_{1-\mathrm{x}}\right) \mathrm{S}$ system in the mentioned $x$ range.

Similarly, the same can be stated for other calculated quantities given that experimental data are available. In Fig. 2 we present our theoretical [33] normed abundances $P_{M} / P_{\text {norm }}$ as a function of the cluster size $M$ together with different measurements of small carbon clusters, obtained

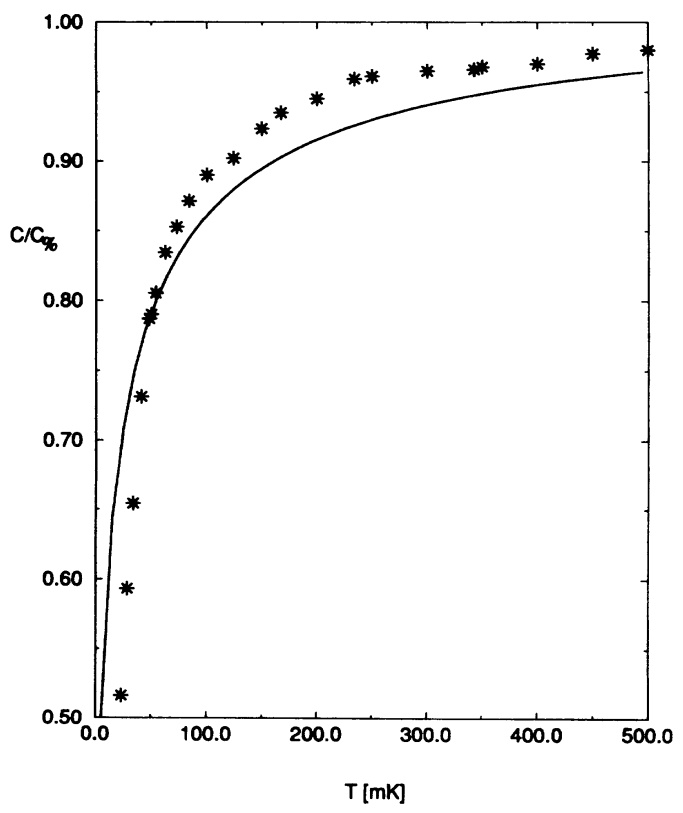

FIG. 1. The temperature dependence of the normalized Curie constant (solid line) is compared with measured data (stars) from Ref. 6 for $\left(\mathrm{Eu}_{x} \mathrm{Sr}_{1-x}\right) \mathrm{S}, x=0.025$. under various circumstances: $C_{M}^{+}, C_{M}^{-}$measurements obtained via sublimation [19], sputtering [20] with $\mathrm{Ar}^{+}$or $\mathrm{Cs}^{+}$, and laser evaporation $[21,22]$. The differences in electron affinities $[8,16]$ account for the odd-even abundances alternations seen in the experimental data. The theoretical results are based only on short-range conditions and statistics, and do not contain concrete information on the carbon chemistry or interaction potential. The differences, relative to measurements, increase around $M=9$, exactly in the region where experiments have indicated nonlinear cluster emergence $[15,17]$. Our model in the low $T$ limit gives [31] $P_{3} / P_{5}=A_{1} / A_{3}=10.0$, which is remarkably close to the measured 11 carbon star [3] value. The calculated $\bar{M}$ is $\sim 2.7$ at low $T$, and, slowly decreasing with increasing $T$, can be compared to both experimental and Monte Carlo (MC) data. The experimental data $[3,19,20]$, where $\bar{M} \sim 2-3$ was measured, confirm the calculated value. In the case of the hard dipolar spheres, the comparison between the calculated $\bar{M}$ with the MC data [10] is presented in Fig. 3, as a function of the concentration $\bar{\rho}$. The MC data fitted in Fig. 3 are specific for chainlike clusters, deduced in the short-range regime [34], at fixed high temperature.

In conclusion, we have presented the first exact solution for a cluster growth model, describing chainlike histories, demonstrating the feasibility of a generic cluster law on a large spectrum of physical systems.

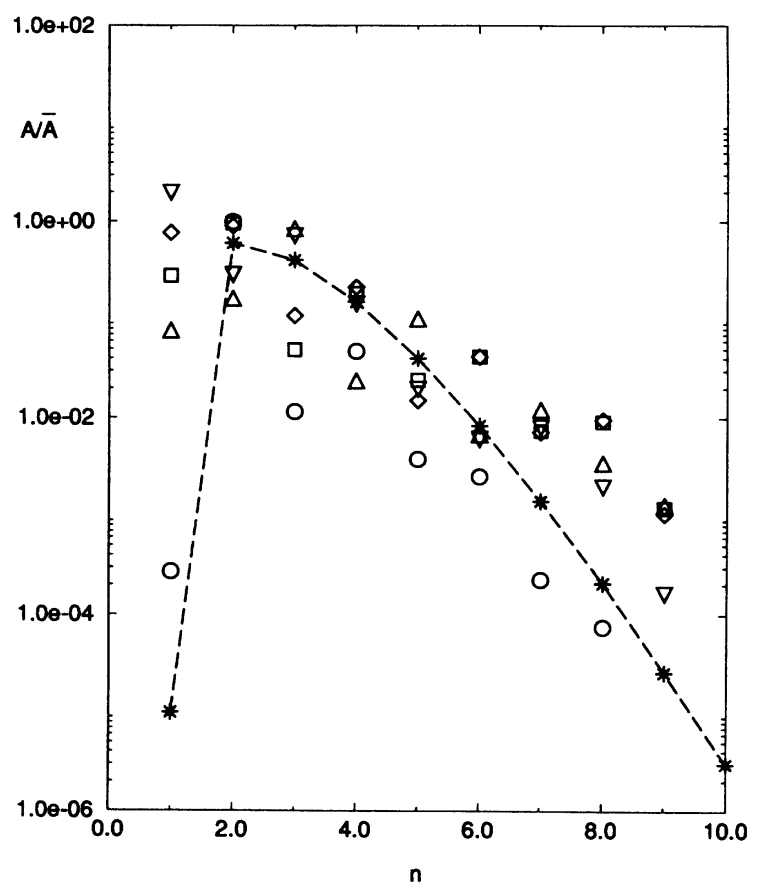

FIG. 2. Normalized carbon cluster abundances as a function of the cluster size $M$ (stars, the long dashed curve is guide for the eye). The experimental data were taken from Ref. 19 (circles); Ref. 20 with Ar (squares) and with Cs (diamonds) sputtering; Ref. 21 (triangles up); and Ref. 22 (triangles down). 


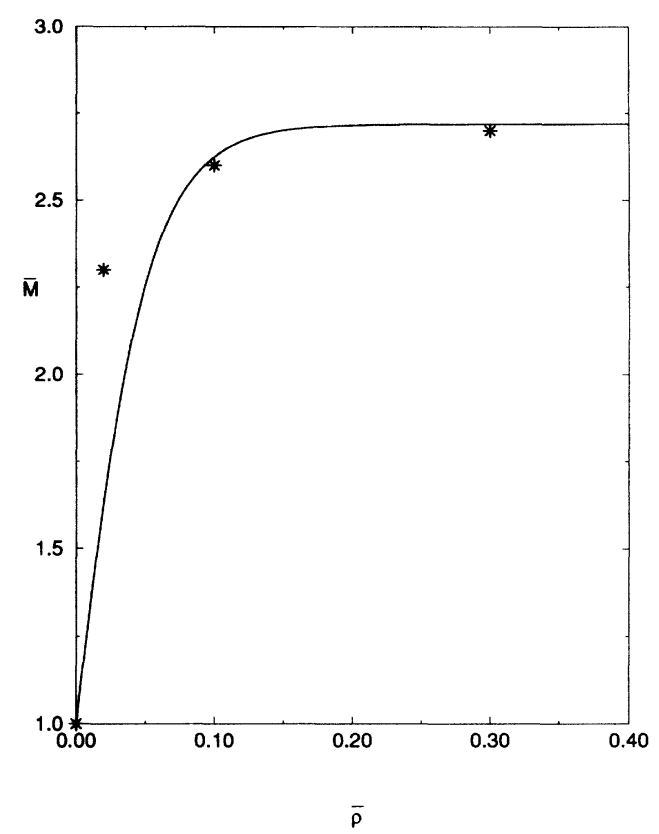

FIG. 3. Average cluster size $\bar{M}$ in function of concentration at fixed temperature (solid line) in comparison with Monte Carlo data (stars) from Ref. 10 for hard dipolar spheres.

For Zs.G., research was supported by the OTKA-T-013952 and MKM $14325 \mathrm{~K}+\mathrm{F}$ contracts. M.G. acknowledges the hospitality of Aspen Center for Physics, where part of this work was done.

[1] Clustering Phenomena in Atoms and Nuclei edited by M. Brenner, T. Lonnroth, and F. B. Malik (SpringerVerlag, Berlin, 1992).

[2] S. G. Kim and D. Tomanek, Phys. Rev. Lett. 72, 2418 (1994).

[3] K. W. Hinkle, J. J. Keady, and P. F. Bernath, Science 241, 1319 (1988); 244, 562 (1989).

[4] W. A. Heer, Rev. Mod. Phys. 65, 611 (1993).

[5] A. A. Abrikosov, Adv. Phys. 29, 869 (1980).

[6] G. Eiselt, J. Kotzler, H. Maletta, D. Staufer, and K. Binder, Phys. Rev. B 19, 2664 (1979).

[7] C.Z. Wang, K. M. Ho, and C. T. Chan, Phys. Rev. B 47, 14835 (1993).

[8] For a review, see W. Weltner, Jr. and R. J. van Zee, Chem. Rev. 89, 1713 (1989).

[9] J. Kotzler and G. Eiselt, Phys. Rev. B 25, 3207 (1982).

[10] J. J. Weis and D. Levesque, Phys. Rev. Lett. 71, 2729 (1993).

[11] G. von Helden, N.G. Gotts, and M. T. Bowers, Nature (London) 363, 60 (1993).

[12] T. W. Ebbesen, J. Tabuchi, and K. Tanigaki, Chem. Phys. Lett. 191, 336 (1992).
[13] K. S. Pitzer and E. Clementi, J. Am. Chem. Soc. 81, 4477 (1959).

[14] A buffer gas supports cluster formation [8].

[15] G. von Helden, P. R. Kemper, N.G. Gotts, and M.T. Bowers, Science 259, 1300 (1993).

[16] D. W. Arnold, S. E. Bradforth, T. N. Kitsopoulos, and D. M. Neumark, J. Chem. Phys. 95, 8753 (1991).

[17] G. von Helden, M.T. Hsu, P.R. Kemper, and M. T. Bowers, J. Chem. Phys. 95, 3835 (1991).

[18] J.R. Heath, R. A. Sheeks, A. L. Cooksky, and R. J. Saykally, Science 249, 895 and 897 (1990).

[19] R. E. Honig, J. Chem. Phys. 22, 124 (1954).

[20] S. N. Schaner, P. Williams, and R. N. Compton, Phys. Rev. Lett. 65, 625 (1990).

[21] J. Berkowitz and W. A. Chupka, J. Chem. Phys. 40, 2735 (1964).

[22] P.P. Radi, T. L. Bunn, P. R. Kemper, M. E. Molchan, and M. T. Bowers, J. Chem. Phys. 88, 2809 (1988).

[23] J. R. Chelikowsky, Phys. Rev. Lett. 67, 2970 (1991).

[24] The average interelement distance $r_{0}$ is much larger than the effective interaction length $l$.

[25] H. G. Bohn, W. Zinn, B. Dorner, and A. Kollmar, Phys. Rev. B 22, 5447 (1980).

[26] J. J. Weis, D. Levesque, and G. J. Zarragoicoechea, Phys. Rev. Lett. 69, 913 (1992).

[27] R. E. Rosenweig, Sci. Am. 10, 124 (1982).

[28] C. F. Hayes and S. R. Hwang, J. Colloid Interface Sci. 60, 443 (1977).

[29] J. Elezgaray, J. F. Muzy, F. Argoul, and A. Arneodo, Phys. Rev. Lett. 71, 2425 (1993).

[30] S. Chandrasekhar, Rev. Mod. Phys. 15, 1 (1943).

[31] If $\eta=k_{B} T, m_{\mathcal{M}} \rightarrow \infty$ corresponds to low $T$ limit at $n_{e}=$ const, where $P_{M}=A_{M-2}, M \geq 2$, and $P_{M=0}=0$.

[32] We consider $\left.J(r)=J_{0} \exp -\gamma r / a\right)$, a being the lattice constant of the conventional fcc cell and $J_{0}=0.44 k_{B}$ fixes the order of magnitude [25]. The $n$th neighboring distance in a fcc lattice is $r_{n}=(n / 2)^{1 / 2} a$. With $\gamma=3$, we obtain $J\left(r_{4}\right)=0.014$ and $J\left(r_{5}\right)=0.0087$. In accordance with the measured values [25] of $J\left(r_{4}\right)=0.014$ and $J\left(r_{5}\right)=0.008, m_{\mathcal{M}}$ is determined from $k_{B} T=J_{0} S(S+$ 1) $\times \exp \left(-\gamma m_{\mathcal{M}} / a\right)$, where $S=7 / 2$ for $\mathrm{Eu}$.

[33] The experimental abundances ratios were deduced from signal intensity ratios. In order to minimize in the normalization factor the effect of differences in the electron affinities $[8,16]$ for odd-even $M$ alternations, we considered $P_{\text {norm }}=P_{2}+P_{3}$. The experimental conditions satisfy Ref. [24], e.g., for Honig [19] $T=2400 \mathrm{~K}$, and $n_{e} \sim 6.1 \times 10^{12}$ particles $/ \mathrm{cm}^{3} \ll 10^{19}$ particles $/ \mathrm{cm}^{3} \sim$ $10^{-3} \mathrm{~g} / \mathrm{cm}^{3}$ Ref. [13]. The theoretical curve is for $m_{\mathcal{M}} \rightarrow \infty$.

[34] The high $T, T^{*}=k T \sigma^{3} / \mu^{2} \gg 0.15$ and low $\rho, \rho^{*}=$ $n_{e} \sigma^{3}<0.4$ define the short-range regime, so $T^{*}=0.25$ was used as MC data. We have $K=\exp \left(-\bar{\alpha} \rho^{*}\right)$, where $\bar{\alpha}=(4 \pi / 3)\left(m_{\mathcal{M}} / \sigma\right)^{3}$. Because of the strong $U_{c}$ energy, $m_{\mathcal{M}} / \sigma=2[10], \bar{M}=\overline{n_{e}}, \bar{\rho}=\rho^{*}$, and $\sigma$ the hard sphere diameter. 